\title{
Aplicativos de celular na prevenção do comportamento suicida*
}

\author{
Sarah Soares Barbosa ${ }^{1}$ \\ (D) https://orcid.org/0000-0002-6847-3942 \\ Jeferson Rodrigues ${ }^{2}$ \\ (D) https://orcid.org/0000-0002-8612-9088 \\ Gustavo Ferreira Guimarães ${ }^{3}$ \\ (D) https://orcid.org/0000-0003-0111-9663 \\ Sara Mendes Boeira Lopes² \\ (D) https://orcid.org/0000-0002-0360-0234
}

Objetivo: identificar aplicativos de celulares utilizados na prevenção e/ou apoio à pessoa com comportamento suicida publicados em produções científicas internacionais e suas funcionalidades. Método: trata-se de uma revisão integrativa da literatura que seguiu o método de Ganong. Foi realizada uma adaptação de método em que foram incluídos critérios de inclusão e exclusão para os aplicativos de celular encontrados nas produções. Foram selecionados os aplicativos e os mesmos foram analisados individualmente para serem mapeadas as suas funcionalidades. Resultados: foram estudados sete aplicativos encontrados a partir da revisão de literatura. Foram mapeadas 71 funcionalidades. Pode-se avaliar que ainda não existem métodos específicos e protocolos para o desenvolvimento de aplicativos para a prevenção do comportamento suicida. Conclusão: foi possível conhecer quais os aplicativos existentes que contribuem na prevenção do comportamento suicida, bem como suas principais funcionalidades. Vê-se a necessidade de que estudos sejam feitos para avaliar o impacto desses aplicativos na prevenção do comportamento suicida, possibilitando o desenvolvimento de pesquisas e dispositivos que previnam o comportamento suicida no Brasil.

Descritores: Suicídio; Aplicativos Móveis; Saúde Mental; Tecnologia.

\section{Como citar este artigo}

Barbosa SS, Rodrigues J, Guimarães GF, Lopes SMB. Mobile applications in the prevention of suicidal behavior. SMAD, Rev Eletrônica Saúde Mental Álcool Drog. 2020;16(4):100-108. doi: https://dx.doi.org/10.11606/issn.1806-6976.smad.2020.167062 


\section{Mobile applications in the prevention of suicidal behavior}

Objective: to identify cell phone applications used in the prevention and/or support of suicidal behavior published in international scientific productions and their functionalities. Method: this is an integrative review of the literature that followed the Ganong method. An adaptation of the method was made in which inclusion and exclusion criteria were included for the cellular applications found in the productions. The applications were selected and analyzed individually to map their functionalities. Results: seven applications found from the literature review were studied. 71 functionalities were mapped. It can be evaluated that there are still no specific methods and protocols for the development of applications for the prevention of suicidal behavior. Conclusion: it was possible to know which existing applications contribute to the prevention of suicidal behavior, as well as their main functionalities. It is necessary that studies are done to evaluate the impact of these applications in the prevention of suicidal behavior, enabling the development of research and devices that prevent suicidal behavior in Brazil.

Descriptors: Suicide; Mobile Applications; Mental Health; Technology.

\section{Aplicaciones de celular en la prevención del comportamiento suicida}

Objetivo: identificar aplicaciones de celulares utilizados en la prevención y/o apoyo a la persona con comportamiento suicida publicados en producciones científicas internacionales y sus funcionalidades. Método: se trata de una revisión integrativa de la literatura que siguió el método de Ganong. Se realizó una adaptación de método donde se incluyeron criterios de inclusión y exclusión para las aplicaciones de celular encontradas en las producciones. Se seleccionaron las aplicaciones y los mismos fueron analizados individualmente para asignar sus características. Resultados: se estudiaron 7 aplicaciones encontradas a partir de la revisión de literatura. Se asignaron 71 funcionalidades. Se puede evaluar que aún no existen métodos específicos y protocolos para el desarrollo de aplicaciones para la prevención del comportamiento suicida. Conclusión: fue posible conocer cuáles las aplicaciones existentes que contribuyen en la prevención del comportamiento suicida, así como sus principales funcionalidades. Se ve la necesidad de que los estudios se realicen para evaluar el impacto de estas aplicaciones en la prevención del comportamiento suicida. Habilitando el desarrollo de investigaciones y dispositivos que previenen el comportamiento suicida en Brasil.

Descriptores: Suicidio; Aplicaciones Móviles; Salud Mental; Tecnología. 


\section{Introdução}

O suicídio é uma das principais causas de morte no mundo, principalmente entre os jovens, e é configurado como um problema grave de saúde pública(1). Segundo a Organização Mundial da Saúde (OMS), a estimativa é que, no ano de 2012, tenha havido cerca de 800 mil mortes por suicídio em algum lugar do mundo, ou seja, uma morte a cada 45 segundos(2).

No fim dos anos 1960, a OMS definiu o comportamento suicida como "multifatorial, multideterminado e transacional", que acontece por fatores complexos, mas identificáveis ${ }^{(2)}$. Entende-se, dessa forma, que não existe uma única causa para o suicídio, pois o mesmo pode ser resultado de uma combinação de fatores pessoais, sociais e de saúde da pessoa(3). O comportamento suicida, por sua vez, é o ato por meio do qual a pessoa causa lesões a si mesma, independendo de intenção letal e da motivação. Pode ser definido como uma continuidade entre pensamentos autodestrutivos por meio de gestos e ameaças e tentativas de suicídio e, finalmente, o suicídio em $\mathrm{si}^{(2)}$.

Ainda que as razões para o suicídio sejam complexas e multifatoriais, identificar o comportamento suicida é fundamental para que este possa ser prevenido. Sabe-se que $66 \%$ das pessoas que cometeram suicídio procuraram atendimento em algum serviço de saúde no mês anterior à sua morte, entretanto, a probabilidade de completar o ato suicida não foi detectada pelos profissionais que realizaram seu atendimento( ${ }^{(3)}$.

A prevenção do suicídio é um desafio que exigirá abordagens sistêmicas, com estratégias de saúde pública e rastreio de pessoas, buscando realizar intervenções, tanto no acompanhamento das pessoas com risco de suicídio, como dos sobreviventes e dos enlutados por uma perda pelo suicídio(3). Nesse sentido, estudiosos do campo do suicídio e da prevenção do suicídio consideram que as lacunas dos serviços de saúde podem ser preenchidas por meio dos avanços tecnológicos e também que são necessárias maiores investigações acerca do tema para que se compreendam quais são as melhores práticas para prevenir, possibilitando salvar mais vidas ${ }^{(4)}$.

Nos Estados Unidos, as Organizações de Serviços Humanos (HSO) reconhecem que os aplicativos podem ser seus aliados na tentativa de lidar com questões sociais e individuais, abrindo um novo e importante canal por meio das tecnologias. Para as HSOs, o uso de tecnologias, entre elas, os aplicativos, pode ser um componente valioso da prestação de serviços ${ }^{(4)}$. Sabe-se que, de fato, a compreensão das melhores práticas para os aplicativos e tecnologias nesse âmbito ainda não está disponível na literatura e, também, que as publicações acerca do tema são escassas e, muitas vezes, inespecíficas ${ }^{(4)}$, o que pode desacelerar o processo de criação de novas tecnologias e/ou medidas de prevenção do comportamento suicida.

Além disso, para a OMS, as intervenções via aplicativos podem ser úteis em países em desenvolvimento onde o apoio às pessoas com risco de suicídio pode ser limitado. Desse modo, faz recomendações acerca do uso de dispositivos móveis, considerando estes uma opção para apoio e tratamento das pessoas em risco de suicídio, uma vez que se sabe que os aplicativos de celulares têm potencial de atingir um grande número de pessoas por meio do uso de dispositivos ${ }^{(5)}$.

Em dezembro de 2019, o mundo assistiu aos primeiros casos de infecção por um novo Coronavírus, o Severe Acute Respiratory Syndrome Coronavirus 2 (Sars-Cov-2), causador da Coronavirus Disease 2019 $($ COVID-19)(6). Devido à disseminação global do vírus, a OMS declara a pandemia de COVID-19 e orienta a população em geral a realizar o distanciamento social para evitar a transmissão(7). Diversas restrições ao funcionamento do comércio, instituições de ensino e áreas de lazer foram criadas pelas autoridades para diminuir o contágio e evitar a sobrecarga dos sistemas de saúde. Neste contexto, o tempo de utilização dos smartphones teve crescimento acentuado e, em diversos locais, a realização de teleconsultas foi uma das medidas propostas, demonstrando a inegável relevância dos aparelhos no cotidiano da população(8).

Devido a esses acontecimentos, apresenta-se um novo cenário, que permite maior contato com os usuários sem a necessidade de encontro físico com o profissional, ampliando as possibilidades de atuação dos serviços de saúde, que podem cuidar do usuário independentemente de limitações geográficas ${ }^{(9)}$, considerando a possibilidade de aplicativos que unam atendimento com profissionais de saúde síncronas e orientações pré-escritas ou gravadas. Além de extinguir as barreiras de acesso físicas, é possível contornar a questão dos horários de atendimento, possibilitando cuidado 24 horas, sete dias por semana(8).

Sabe-se, considerando a urgência do usuário com comportamento suicida em crise e a necessidade de acompanhamento, que o uso de aplicativos que previnem e oferecem apoio à pessoa com comportamento suicida pode ser de grande valia, principalmente no contexto da pandemia de COVID-19.

Em estudo recente conduzido no Brasil, os resultados demonstraram efeitos na saúde mental dos participantes durante a pandemia de COVID-19 que se relacionaram com o aumento da exposição dos mesmos a notícias e informações sobre os óbitos e sequelas da doença, o que possibilitaria um aumento na demanda dos serviços de saúde mental(9). Diante deste achado, a utilização de aplicativos poderia facilitar o acesso aos usuários, informar conhecimento científico de forma a reduzir os possíveis danos psicológicos e orientar 
sobre os locais que fornecem cuidados emergenciais de atenção psicológica. Ressalta-se também que, diante da realidade de crise social e da urgência no atendimento à saúde mental, uma das possibilidades de grande valia que pode ser realizada é que os profissionais de saúde realizem intervenções e atendimentos on-line. Existe também a necessidade de instrumentalizar os profissionais de saúde que irão atender estes pacientes e capacitar os mesmos para a abordagem, focando no atendimento aos grupos com maior vulnerabilidade ${ }^{(10)}$.

Diante do exposto, o objetivo deste estudo foi identificar os aplicativos de celulares utilizados na prevenção e/ou apoio à pessoa com comportamento suicida publicados em produções científicas internacionais e quais suas funcionalidades.

\section{Método}

Trata-se de um estudo desenvolvido a partir de uma Revisão Integrativa da Literatura, entendida como um método de pesquisa que possibilita a organização sistemática e síntese de evidências disponíveis acerca do tema a ser estudado. A análise dos estudos incluídos nesta revisão foi realizada de forma dinâmica e incluiu estudos teóricos, empíricos e permitiu reuni-los para que fosse construída uma conclusão geral a partir dos resultados de cada estudo(11).

Para o desenvolvimento deste estudo, elaborou-se um protocolo adaptado para a revisão integrativa da literatura e foram seguidas as etapas da revisão(11-12): seleção da pergunta de pesquisa; definição dos critérios de inclusão e exclusão dos estudos; seleção da amostra; representação dos estudos selecionados em formato de tabelas, considerando as características em comum; análise crítica dos resultados, identificando diferenças e conflitos; discussão e interpretação dos resultados; apresentação, de forma clara, da evidência encontrada. Adotaram-se as recomendações PRISMA (Preferred Reporting Items for Systematic reviews and Meta-Analyse) ${ }^{(13)}$ - conforme fluxograma apresentado nos resultados e preconizado em estudos de revisão nos modelos baseados em evidência.

Os artigos foram identificados a partir de busca bibliográfica realizada no período de dezembro de 2016 a julho de 2020 nas bases de dados a seguir: MEDLINE/ PubMed $^{\circledR}$ (via National Library of Medicine); SCOPUS ${ }^{\circledR}$ (Elsevier); Web of Science ${ }^{\circledR}$ (Thomson Reuters Scientific); PsycINFO ${ }^{\circledR}$ (APA); CINAHL ${ }^{\circledR}$ (EBSCO Host); Academic Search Premier (EBSCO Host); LILACS ${ }^{\circledR}$ (via Biblioteca Virtual em Saúde).

A captação dos artigos foi realizada em cada base de dados de forma individual, não sendo prédefinida uma base para início e/ou término, ou seja, a busca deu-se de forma aleatória. Todos os trabalhos encontrados, atendendo os critérios de busca, foram submetidos à próxima etapa do protocolo.
Foram incluídos, no estudo, os artigos publicados em inglês, espanhol e português, não delimitando data de publicação, que apresentassem resumos e informações sobre a utilização de aplicativos de celular para a prevenção do suicídio.

As estratégias de busca utilizadas foram:

1. MEDLINE/PubMed: ("Suicide"[Mesh] OR suicide*) AND ("Mobile Applications" OR "Mobile Application" OR "app" OR "apps") AND (phone* OR mobile* OR cellphone*);

2. SCOPUS: suicide* AND ("Mobile Applications" $O R$ "Mobile Application" OR "app" OR "apps") AND (phone* OR mobile* OR cellphone*);

3. Web of Science: suicide* AND ("Mobile Applications" $O R$ "Mobile Application" $O R$ "app" $O R$ "apps") AND (phone* OR mobile* $O R$ cellphone*);

4. PsycINFO: suicide* AND ("Mobile Applications" $O R$ "Mobile Application" OR "app" OR "apps") AND (phone* OR mobile* OR cellphone*);

5. CINAHL: suicide* AND ("Mobile Applications" $O R$ "Mobile Application" $O R$ "app" OR "apps") AND (phone* OR mobile* OR cellphone*);

6. Academic Search Premier: suicide* AND ("Mobile Applications" OR "Mobile Application" $O R$ "app" OR "apps") AND (phone* OR mobile* OR cellphone*);

7. LILACS: suicid* AND ("Mobile Applications" $O R$ "Mobile Application" $O R$ "app" $O R$ "apps" $O R$ aplicativ* AND phone* OR mobile* $O R$ cellphone* OR celular* OR move*).

A captação e organização dos estudos foram realizadas a partir de pastas de arquivos nomeadas com os títulos das bases de dados. Os estudos foram selecionados e armazenados a partir da leitura dos títulos e resumos para que fossem determinados os objetos de análise aprofundada. Essa primeira etapa é denominada "primeira peneira" e possibilitou reconhecer os estudos alvo de análise conforme o escopo deste protocolo. A partir dessa primeira etapa, como indica a Revisão Integrativa(11-12), os dados foram sistematizados em tabelas com respectiva leitura criteriosa dos estudos e organização dos dados por meio de numeração e título.

Assim, a busca inicial apresentou 89 publicações, sendo que, após leitura de título e resumo, foram excluídos estudos em duplicidade nas diferentes bases de dados, artigos indisponíveis para download e os estudos que não respondiam à questão norteadora da pesquisa. Nesta etapa, foram selecionados 73 artigos para a leitura aprofundada na íntegra. Destes, 66 estudos foram excluídos por não responderem ao escopo da pesquisa, resultando em uma amostra final de sete artigos para análise. 


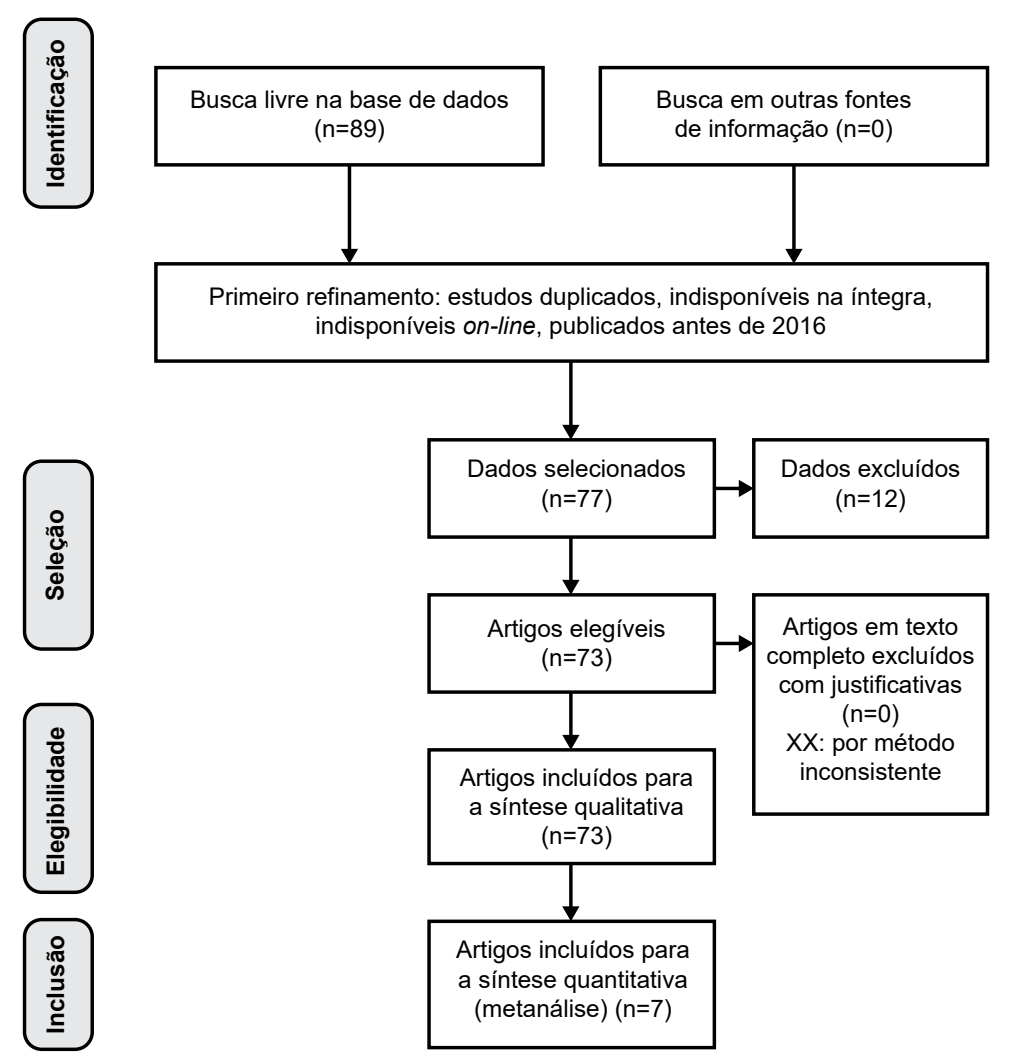

Fonte: Prisma (2020) $)^{(11)}$

Figura 1 - Fluxograma de Revisão Integrativa da Literatura

Foi realizada releitura dos materiais préselecionados com avaliação crítica e sistematização dos dados em categoria, procedendo-se à Análise do Conteúdo(14), definida como um conjunto de técnicas de análise das comunicações que utiliza um sistema objetivo de descrição dos conteúdos. As informações extraídas dos estudos foram: título do artigo; autores; ano de publicação; referência completa do artigo; objetivo da pesquisa; metodologia; reflexão dos artigos a respeito do tema; aplicativos analisados no estudo e resultados da pesquisa.

\section{Resultados}

Foram selecionados sete artigos que atenderam aos critérios de inclusão propostos para esta Revisão Integrativa. As bases de dados que tiveram o maior número de publicações incluídas foram a Academic Search Premier e a SCOPUS, com três artigos. Informa-se que $100 \%$ dos estudos selecionados foram publicados no idioma inglês e o período de publicação deu-se entre 2013 e 2017, sendo que três estudos foram publicados em 2017, um, em 2016, um, em 2014 e dois, em 2013. Os estudos foram publicados em quatro países: Austrália (3); Estados Unidos da América (2); Bélgica (1) e Dinamarca (1). Na Figura 2, são apresentados os artigos selecionados.

Quanto aos tipos de pesquisa, A1 tratava-se de estudo de metodologia descritiva; A2, A3 e A4 eram estudos qualitativos - sendo A3 um ensaio clínico randomizado. $\mathrm{O}$ artigo $\mathrm{A} 1$ considerou como diferentes os aplicativos que eram desenvolvidos para as duas plataformas de desenvolvimento (IOS $₫$ e Android $^{\circledR}$ ), tendo um total de 49 aplicativos; $A 2$ apresentou 27 aplicativos sem especificar diferenças entre as plataformas de desenvolvimento; A3 apresentou apenas um aplicativo já que trazia, em seu estudo, a proof-of-concept da utilização do app visando a avaliar sua viabilidade em uma amostra de veteranos de guerra americanos. 0 artigo A4 não apresentou o nome do aplicativo, uma vez que o estudo abordou o desenvolvimento do $a p p$ para avaliar a efetividade do mesmo para a prevenção do suicídio em jovens aborígenes australianos e está em fase de recrutamento para testes. $O$ artigo A5 trouxe o nome do aplicativo e discorreu sobre sua criação, dois testes de uso e o lançamento em setembro de 2015. Em aproximadamente seis meses após o lançamento do aplicativo do artigo A5, este teve cerca de 1500 downloads e diversas avaliações positivas e, em 2017, foi atualizado para a inclusão de novas funcionalidades. $\mathrm{O}$ artigo A6 é o relato do teste com oito comunidades indígenas do aplicativo, que tem o desenvolvimento relatado no artigo A4 e mostra resultados expressivos na diminuição das taxas de suicídio e de sofrimento psíquico agudo, necessitando de intervenção por profissionais de saúde nestas comunidades. O A7 é um estudo clínico randomizado que incluiu 546 participantes em que 
metade destes recebeu acesso ao aplicativo e a outra metade teve acesso ao conteúdo do aplicativo, porém, na versão física em papel, após seis semanas de uso do aplicativo, o mesmo foi avaliado pelos usuários de teste.
Os dados coletados demonstraram maior efetividade do aplicativo pela disponibilidade instantânea do mesmo e novas funcionalidades seriam implementadas após avaliação e sugestão dos usuários.

\begin{tabular}{|c|c|}
\hline Identificação Artigo & Referência Completa \\
\hline A1 & $\begin{array}{l}\text { Larsen ME; Nicholas J; Christensen H. A Systematic Assessment of Smartphone Tools for Suicide Prevention. Public Library } \\
\text { of Science, PloS One. [Internet]. 2016;11( 4):1-14. Public Library of Science (PLoS). [cited Jan } 27 \text { 2017]. Available from: } \\
\text { <http://journals.plos.org/plosone/article?id=10.1371/journal.pone.0152285>. DOI: 10.1371/journal.pone.0152285(1) }\end{array}$ \\
\hline $\mathrm{A} 2$ & $\begin{array}{l}\text { Aguirre RTP, McCoy, MK, Roan M. Development Guidelines from a Study of Suicide Prevention Mobile Applications (Apps). } \\
\text { J Technol Hum Serv. [Internet]. 2013; 31(3), 269-93. Available from: https://www.tandfonline.com/doi/abs/10.1080/15228835 } \\
.2013 .814750 \text { DOI: } 10.1080 / 15228835.2013 .814750^{(4)}\end{array}$ \\
\hline A3 & $\begin{array}{l}\text { Bush NE, Dobscha SK, Crumpton R, Denneson LM, Hoffman JE, Crain A et al. A Virtual Hope Box Smartphone App as an } \\
\text { Accessory to Therapy: Proof-of-Concept in a Clinical Sample of Veterans. Suicide And Life-threatening Behavior. [Internet]. } \\
\text { 2014;45(1):1-19. [cited Jan } 27 \text { 2017]. Available from: <http://onlinelibrary.wiley.com/doi/10.1111/sltb.12103/abstract>. DOI: } \\
\text { http://dx.doi.org/10.1111/sltb.12103 } 1 \text { : } 15 \text {. }\end{array}$ \\
\hline A4 & $\begin{array}{l}\text { Shand FL, Ridani R, Tighe J, Christensen H. The effectiveness of a suicide prevention app for indigenous Australian youths: } \\
\text { study protocol for a randomized controlled trial. Trials. [Internet]. 2013;14(1):3961-403. [cited Jan 27 2017]. Available from: } \\
\text { <https://www.ncbi.nlm.nih.gov/pmc/articles/PMC4222891/>. DOI: http://dx.doi.org/10.1186/1745-6215-14-396 (16) }\end{array}$ \\
\hline A5 & $\begin{array}{l}\text { Auwels K; Aerts S; Muijzers E; Jaegere E, Heeringen K, Portzky G. BackUp: development and evaluation of a smart-phone } \\
\text { application for coping with suicidal crises. Plos One. [Internet] 2017;12( 6):1-16. [cited Jan } 27 \text { 2017]. Available from: https:// } \\
\text { journals.plos.org/plosone/article?id=10.1371/journal.pone.0178144. DOI: 10.1371/journal.pone.0178144(17) }\end{array}$ \\
\hline A6 & $\begin{array}{l}\text { Tighe J, Shand F, Ridani R, Mackinnon A, La Mata N de, Christensen H. Ibobbly mobile health intervention for suicide } \\
\text { prevention in Australian Indigenous youth: a pilot randomised controlled trial. Bmj Open. [Internet] 2017;7(1):013518- } \\
013528 \text { [cited Aug } 032020 \text { ]. Available from: https://bmjopen.bmj.com/content/7/1/e013518. DOI:. http://dx.doi.org/10.1136/ } \\
\text { bmjopen-2016-013518 }{ }^{(18)}\end{array}$ \\
\hline A7 & $\begin{array}{l}\text { Andreasson K, Krogh J, Bech P, Frandsen H, Buus N, Stanley B et al. MYPLAN-mobile phone application to manage crisis } \\
\text { of persons at risk of suicide: study protocol for a randomized controlled trial. Trials. [Internet] 2017;18(1):1-7. [cited Aug 03 } \\
\text { 2020]. Available from: https://www.ncbi.nlm.nih.gov/pmc/articles/PMC5387214/ DOI: http://dx.doi.org/10.1186/s13063-017- } \\
1876-9^{(19)}\end{array}$ \\
\hline
\end{tabular}

Figura 2 - Artigos Encontrados na Revisão Integrativa da Literatura

\section{Discussão}

O uso das Tecnologias da Informação e Comunicação (TICs), no que se refere aos apps no campo da prevenção do comportamento suicida, mostrou-se amplo. Sobremaneira, sabe-se que a prevenção do comportamento suicida envolve um conjunto de estratégias a serem ofertadas ao usuário para que este acesse em momentos de sofrimento ou preocupação excessiva para ele. Para tanto, considera-se a possibilidade de desenvolver um aplicativo para celular com o maior número de recursos adequados possível, que seja baseado em estudos ou experiências clínicas sobre o tema, bem como adequado à realidade cultural do local onde se pretende utilizá-lo. Nesse aspecto, destaca-se que existem diferenças nos tipos de intenção suicida entre os países ou até mesmo entre regiões de um mesmo país, e essas culturas distintas possuem papel importante e devem ser consideradas pelos formuladores de estratégias de prevenção e de políticas públicas ${ }^{(19)}$.

Desse modo, entender os significados culturais do suicídio, seus fatores de risco e proteção em diferentes culturas e até mesmo a forma como o suicídio é compreendido por membros de uma cultura específica é importante para o desenvolvimento das intervenções para essas pessoas etnicamente diversas e com diferentes crenças sobre o comportamento suicida(20).

Portanto, uma equipe com conhecimento e preparo técnico relacionados ao tema de prevenção do comportamento suicida em apps parece fundamental, uma vez que as ferramentas de apoio ao usuário devem ser desenvolvidas com embasamento teórico quanto ao que realmente poderá fornecer o suporte necessário para que o usuário se mantenha seguro para recursos que minimizem o comportamento suicida.

Assim, infere-se que os aplicativos móveis para a promoção, educação e cuidados com a saúde devem ser baseados em teorias e evidências científicas, objetivando garantir que as informações neles contidas sejam sérias, de conteúdos embasados pela literatura, para uma mudança, de fato, no comportamento e evitando deixar os usuários desprotegidos ou até mesmo colocálos em risco no seu tratamento de saúde ${ }^{(21)}$. Nesse aspecto, reforça-se que, além de não terem sido encontrados aplicativos de celular para a prevenção do comportamento suicida no Brasil nesta revisão, também não foram encontrados estudos no Brasil que contemplassem testes clínicos ou validações de aplicativos de celular em desenvolvimento para este fim, o que mostra uma lacuna neste campo do conhecimento.

Ainda nesse sentido, lembra-se que, além do embasamento teórico e científico no desenvolvimento dos apps, é fundamental que eles sejam testados quanto à sua usabilidade e eficácia, já que, se desenvolvidos adequadamente, atingem grande público e passam a representar uma ferramenta utilizável em saúde pública. Vê-se que esta situação abre espaço para que profissionais da área da saúde trabalhem em parcerias como desenvolvedores de aplicativos móveis, visando a 
auxiliar na produção de ferramentas com intervenções baseadas em evidências que reduzam riscos, ampliem acesso a serviços e melhorem a inclusão social e a interatividade com o uso de apps ${ }^{(21)}$.

Estudos mostram que há, de fato, falta de provas de utilidade, eficácia e segurança de uso de aplicativos em saúde(21), deixando em aberto as questões sobre a efetividade dos aplicativos já existentes no mercado e podendo, dessa forma, gerar insegurança quanto à indicação de seu uso pelos profissionais de saúde, por exemplo.

Outro estudo trouxe a grande rotatividade dos aplicativos de saúde mental no mercado das app stores. Afirmou-se que, além de oferecerem poucas informações sobre a qualidade do conteúdo do $a p p$, são poucos os que citam a fonte desse conteúdo e que fundamentam sua eficácia(22).

Buscou-se, sabendo das lacunas encontradas, refletir quanto às funcionalidades existentes nos aplicativos vistos nesta revisão, objetivando conhecer quais destas estratégias de prevenção são corroboradas por estudiosos do tema. Sobre os recursos informativos sobre sinais e sintomas psiquiátricos, sabe-se que informar quais são os sinais e sintomas de alerta pode ser fundamental para a proteção de indivíduos em risco. As Diretrizes Nacionais para a Prevenção do Suicídio trazem, em seu artigo segundo: "desenvolver estratégias de informação, de comunicação e de sensibilização da sociedade de que o suicídio é um problema de saúde pública que pode ser prevenido"(23), ou seja, recursos informativos são considerados relevantes para o campo da prevenção e, portanto, devem estar presentes em um aplicativo para a prevenção do comportamento suicida. Os contatos de serviços de saúde e saúde mental são fundamentais, pois profissionais observaram que este preenchimento de informações sobre a rede de apoio do usuário permite que o mesmo tenha uma visão geral das pessoas com quem pode contar para situações críticas, fazendo com que perceba com quem poderá conversar sobre seu sofrimento e seus pensamentos, por exemplo(24).

Os recursos para ajuda on-line/outros recursos on-line são maneiras que permitem, por exemplo, que o usuário atualize e acesse seu plano de segurança onde quer que esteja. Além disso, há de considerar que o uso de aplicativos para celular, que estejam conectados à internet, permite que se apliquem recursos intrínsecos às tecnologias, como a capacidade contínua de transmissão de informações, utilização de elementos multimídia e geolocalização(5). Além disso, vale considerar que soluções em "nuvem" permitem o armazenamento dos dados na internet, podendo melhorar ainda mais as funções do aplicativo(5).

Os recursos com vídeos e imagens, por sua vez, permitem que o usuário armazene itens como músicas favoritas, fotografias de família ou lembranças de bons momentos. O objetivo deste espaço é fornecer um meio de o usuário recordar suas razões para viver em momentos de angústia e desencorajamento, quando pode estar suscetível ao comportamento suicida. A utilização desses elementos também pode servir como recurso para distração, buscando reforçar habilidades de tolerância ao estresse, por exemplo(25).

O Plano de Segurança ou Cartão de Crise é uma já conhecida estratégia no campo de saúde mental, principalmente quando se trata de usuários com risco de autoagressão ou suicídio. Nesse aspecto, sabe-se que, em alguns países, como a Holanda, por exemplo, o plano de crise é executado em organizações que disponibilizam, entre outros recursos, um consultor que se responsabiliza por acompanhar todo o processo com o usuário. Em alguns casos, o plano pode ser acessado na internet, caso permitido pelo usuário, ficando disponível para as entidades e pessoas previamente acordadas ${ }^{(24)}$. Esses recursos são utilizados como diretrizes e parte do tratamento em clínicas de prevenção ao suicídio, e os pacientes são encorajados a desenvolver seus próprios planos de segurança. A principal vantagem do plano de segurança eletrônico, portanto, é que o usuário o levará consigo na maioria das vezes ${ }^{(5)}$.

Salienta-se o forte embasamento teórico e empírico que sustenta os recursos de planos de segurança, uma vez que os mesmos possuem estratégias validadas na prevenção do suicídio em adultos, principalmente nas linhas cognitivo-comportamentais ${ }^{(26)}$. Os recursos para relaxamento, atividades físicas e mindfulness podem ser justificados como parte do conteúdo dos apps, uma vez que estudos demonstraram que a realização de atividades físicas tem impacto positivo na vida das pessoas com sofrimentos psíquicos ${ }^{(24)}$. Indicaram, ainda, que esta pode ser uma ferramenta útil no tratamento das pessoas que experimentaram comportamento suicida no passado, pois os ajuda a adquirir habilidades que Ihes permitirão responder às crises de maneira mais rápida e hábil(23). Quanto ao relaxamento, sabe-se que, quando combinado com outras estratégias de cuidado e tratamento, o mesmo pode colaborar para reduzir os sintomas depressivos e de ansiedade, por exemplo(25).

Assim, entre as categorias de funcionalidades listadas, que podem contribuir para a prevenção do comportamento suicida, pode-se perceber que os recursos de contatos de serviços de saúde e saúde mental; recursos para ajuda on-line/outros recursos on-line e recursos com vídeos e imagens possuem algum embasamento quando comparados às estratégias para a prevenção do comportamento suicida estudadas. Os recursos Plano de Segurança ou Cartão de crise, relaxamento, atividades físicas e mindfulness, em especial, parecem trazer benefícios importantes às pessoas com algum sofrimento mental. Há que se considerar que estas já são estratégias utilizadas independentemente do meio eletrônico e propõem atividades que podem ajudar o usuário a descontrair e mudar o foco da sua atenção para outras $\operatorname{coisas}^{(27)}$. 


\section{Conclusão}

Foi possível identificar quais aplicativos de celular para a prevenção do suicídio estão disponíveis no mercado e quais estão em fase de teste por meio das produções científicas, bem como conhecer quais suas principais funcionalidades e quais são os recursos que se apresentam disponíveis para a prevenção do comportamento suicida. Promover e conectar usuários aos serviços e programas de suporte às pessoas com risco de suicídio é uma estratégia que pode colaborar nos momentos de crise e risco para o comportamento suicida, tendo em vista que esses serviços oferecem apoio especializado ao usuário em sofrimento.

Observou-se que estratégias, como o Plano de Segurança, podem ser úteis no comportamento suicida e possuem fundamento teórico, uma vez que já são amplamente utilizadas nos tratamentos dos usuários de saúde mental com risco para o comportamento suicida. Medidas de meditação e relaxamento também trazem benefícios às pessoas em sofrimento, já que têm capacidade de melhorar sintomas depressivos e ansiedade, refletindo em uma melhora do estado geral das mesmas.

Essas funcionalidades possuem embasamento teórico e empírico e, desse modo, não podem deixar de estar presentes nos aplicativos de celular para a prevenção do comportamento suicida. Entretanto, vê-se que devem ser realizadas pesquisas que avaliem o impacto dos aplicativos de celular na prevenção do comportamento suicida, já que são escassas as produções científicas acerca do tema e, principalmente, a comprovação da eficácia destes para os usuários de saúde mental em momentos de sofrimento.

Além disso, não foram encontrados estudos brasileiros sobre o tema, o que aponta uma lacuna de conhecimento entre os estudiosos acerca do tema no país, bem como não foi encontrado, na busca de aplicativos, algum que tenha sido desenvolvido no Brasil. Desse modo, há que se incentivar a criação de projetos e estudos interdisciplinares que proponham e avaliem o desenvolvimento de pesquisas e dispositivos que previnam o comportamento suicida, colaborando com a produção científica acerca deste tema tão importante e ainda pouco difundido no Brasil. A utilização de smartphones no contexto da pandemia de COVID-19 demonstra o quanto a área da saúde pode se utilizar destes aparelhos para o cuidado com o paciente.

\section{Referências}

1. Larsen ME, Nicholas J, Christensen H. A Systematic Assessment of Smartphone Tools for Suicide Prevention. PloS One. [Internet]. 2016;11(4):1-14. doi: 10.1371/ journal.pone. 0152285.

2. Botega NJ. Crise Suicida: Avaliação e Manejo. Porto Alegre (RS): Artmed; 2015. 302 p.
3. Larsen ME, Cummins N, Boonstra TW, O'Dea B, Tigue J, Nicholas J, et al. The use of technology in Suicide Prevention. Conf Proc IEEE Enfg Med Biol Soc. 2015; 1(1):7316-9. doi: 10.1109/EMBC.2015.7320081.

4. Aguirre RTP, McCoy, MK, Roan M. Development Guidelines from a Study of Suicide Prevention Mobile Applications (Apps). J Technol Hum Serv. [Internet]. 2013; 31(3):269-93. doi: 10.1080/15228835.2013.814750.

5. Larsen JLS, Frandsen H, Erlangsen A. MYPLAN - A Mobile Phone Application for Supporting People at Risk of Suicide. Crisis. [Internet]. 2016; 37(3):236-40. doi: 10.1027/0227-5910/a000371

6. Wang C, Pan R, Wan X, Tan Y, Xu L, Ho CS et al. Immediate Psychological Responses and Associated Factors during the Initial Stage of the 2019 Coronavirus Disease (COVID-19) Epidemic among the General Population in China. Int J Environ Res Public Health. [Internet]. 2020 Mar;17(5):1729-54. doi: http://dx.doi. org/10.3390/ijerph17051729.

7. Li W, Yang, Y, Liu ZH, Zhao YJ, Zhang Q, Zhang L, et al. Progression of mental health services during the COVID19 outbreak in China. Int JBiol Sci. 2020;16(10):1732-8. doi: http://dx.doi.org/10.7150/ijbs.45120

8. Caetano R, Silva AB, Guedes ACCM, Paiva CCN de, Ribeiro GR, Santos DL, et al. Challenges and opportunities for telehealth during the COVID-19 pandemic: ideas on spaces and initiatives in the Brazilian context. Cad Saúde Pública. 2020;36(5):e00088920-e00088936. doi: http://dx.doi.org/10.1590/0102-311x00088920.

9. Duarte MQ, Santo MAS, Lima CP, Giordani JP, Trentini CM. Covid-19 and the impacts on mental health: a sample from Rio Grande do Sul, Brazil. Ciênc Saúde Coletiva. [Internet]. 2020 [cited 2020 Sep 2];25(9). doi: 10.1590/1413-81232020259.16472020.

10. Faro A, Bahiano MA, Nakano TC, Reis C, Silva BFP, Vitti LS. COVID-19 and mental health: the emergence of care. Estud Psicol. 2020;37:e200074. doi: http:// dx.doi.org/10.1590/1982-0275202037e200074.

11. Pompeo DA, Rossi LA, Galvão CM. Revisão integrativa: etapa inicial do processo de validação de diagnóstico de enfermagem. Acta Paul Enferm. 2009;22(4):434-38. doi: $10.1590 /$ S0103-21002009000400014

12. Ganong LH. Integrative reviews of nursing. Rev. Nurs Health. 1987;10(1):1-11. doi: 10.1002/nur.4770100103 13. Moher D, Liberati A, Tetzlaff J, Altman DG. The PRISMA Group (2009). Preferred Reporting Items for Systematic Reviews and Meta-Analyses: The PRISMA Statement. PLoS Med. 2009;6(7): e1000097. doi: 10.1371/journal.pmed1000097

14. Bardin L. Análise de conteúdo. São Paulo (SP): Edições 70; 2011. 229 p.

15. Bush NE, Dobscha SK, Crumpton R, Denneson LM, Hoffman JE, Crain A, et al. A Virtual Hope Box Smartphone App as an Accessory to Therapy: Proof-ofConcept in a Clinical Sample of Veterans. Suicide Lifethreatening Behav. 2014;45(1):1-19. doi: http://dx.doi. org/10.1111/sltb.12103. 
16. Shand FL, Ridani R, Tighe J, Christensen H. The effectiveness of a suicide prevention app for indigenous Australian youths: study protocol for a randomized controlled trial. Trials. 2013;14(1):3961-403. doi: http://dx.doi.org/10.1186/1745-6215-14-396

17. Auwels K, Aerts S, Muijzers E, Jaegere E, Heeringen K, Portzky G. BackUp: development and evaluation of a smart-phone application for coping with suicidal crises. Plos One. [Internet] 2017;12( 6):1-16. doi: 10.1371/ journal.pone. 0178144

18. Tighe J, Shand F, Ridani R, Mackinnon A, La Mata N de, Christensen $\mathrm{H}$. Ibobbly mobile health intervention for suicide prevention in Australian Indigenous youth: a pilot randomised controlled trial. BMJ Open. [Internet] 2017;7(1):013518-013528. doi: http://dx.doi. org/10.1136/bmjopen-2016-013518

19. Andreasson K, Krogh J, Bech P, Frandsen $H$, Buus N, Stanley $B$, et al. MYPLAN -mobile phone application to manage crisis of persons at risk of suicide: study protocol for a randomized controlled trial. Trials. [Internet] 2017;18(1):1-7. doi: http://dx.doi.org/10.1186/ s13063-017-1876-9

20. Freeman A, Mergl R, Kohls E, Székely A, Gusmão $\mathrm{R}$, Arensman $\mathrm{E}$ et al. A cross-national study on gender differences in suicide intent. BMC Psychiatry. 2017;17(1). doi: 10.1186/s12888-017-1398-8.

21. Chu J, Khoury O, Ma J, Bahn F, Bongar B, Goldblum P. An Empirical Model and Ethnic Differences in Cultural Meanings Via Motives for Suicide. J Clin Psychol. [Internet] 2017;73(10):1343-59. doi: 10.1002/ jclp. 22425.

22. Rocha FS, Santana EB, Silva ES, Carvalho JSM, Carvalho FLQ. Uso de apps para a promoção dos cuidados à saúde. STAES 2017. [Internet] 2017;3(1):1-10. [Acesso 24 ago 2020]. Disponível em: <https://revistas. uneb.br/index.php/staes/article/view/3832/2382>.

23. Portaria No 1.876 ( BR). Institui Diretrizes Nacionais para Prevenção do Suicídio, a ser implantadas em todas as unidades federadas, respeitadas as competências das três esferas de gestão. [Internet] 2006[Acesso 24 ago 2020]. Disponível em: <https://www.jusbrasil.com.br/ diarios/687743/pg-65-secao-1-diario-oficial-da-uniaodou-de-15-08-2006>

24. Freitas C. A participação e preparação prévia do usuário para situações de crise mental: a experiência holandesa do plano/cartão de crise e desafios para sua apropriação no contexto brasileiro. In: Vasconcelos
EM, organizador. Abordagens psicossociais: Reforma Psiquiátrica e Saúde Mental na ótica da cultura e das lutas populares. São Paulo: Hucitec; 2008. v. 2, p. 142-70.

25. Motta CCL, Moré CLOO, Nunes CHSS. O atendimento psicológico ao paciente com diagnóstico de depressão na Atenção Básica. Cienc Saúde Coletiva. 2017;22(3):91120. doi: 10.1590/1413-81232017223.27982015

26. Williams JMG, Duggan DS, Crane C, Fennell M. Mindfulness-Based cognitive therapy for prevention of recurrence of suicidal behavior. J Clin Psychol. [Internet] 2005;62(2):201-10. doi: 10.1002/jclp.20223

27. Yatham K, Yoon R, da Silva TL, Ravindran AV. Efficacy of brief interventions for mental illnesses in lowand middle-income countries. Annals Global Health. 2016;82(3):573. doi: 10.1016/j.aogh.2016.04.535.

\section{Contribuição dos autores}

Concepção e planejamento do estudo: Sarah Soares Barbosa, Jeferson Rodrigues e Gustavo Ferreira Guimarães. Obtenção dos dados: Sarah Soares Barbosa, Jeferson Rodrigues, Gustavo Ferreira Guimarães e Sara Mendes Boeira Lopes. Análise e interpretação dos dados: Sarah Soares Barbosa, Jeferson Rodrigues e Gustavo Ferreira Guimarães. Análise estatística: Sarah Soares Barbosa, Jeferson Rodrigues, Gustavo Ferreira Guimarães e Sara Mendes Boeira Lopes. Redação do manuscrito: Sarah Soares Barbosa, Jeferson Rodrigues, Gustavo Ferreira Guimarães e Sara Mendes Boeira Lopes. Revisão crítica do manuscrito: Sarah Soares Barbosa, Jeferson Rodrigues, Gustavo Ferreira Guimarães e Sara Mendes Boeira Lopes.

Todos os autores aprovaram a versão final do texto. Conflito de interesse: os autores declararam que não há conflito de interesse. 